\title{
Huge Brain Cystic Lesions Resulting from Metronidazole-Induced Encephalopathy
}

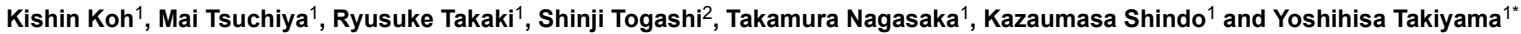

${ }^{1}$ Department of Neurology, Graduate School of Medical Sciences, University of Yamanashi, Japan

${ }^{2}$ Department of Neurology, Kofu Municipal Hospital, Japan

*Corresponding author: Yoshihisa Takiyama, Department of Neurology, Graduate School of Medical Sciences, University of Yamanashi, 1110 Shimokato, Chuo-shi, Yamanashi 409-3898, Japan, Tel: +81 55273 9896; Fax: +81 55273 9896; E-mail: ytakiyama@yamanashi.ac.jp

Received date: January 19, 2016; Accepted date: January 22, 2016; Published date: January 29, 2016

Copyright: @ 2016 Koh K, et al. This is an open-access article distributed under the terms of the Creative Commons Attribution License, which permits unrestricted use, distribution, and reproduction in any medium, provided the original author and source are credited.

\begin{abstract}
Metronidazole-induced encephalopathy (MIE) is a rare clinical condition resulting from long-term use of metronidazole. The symptoms and brain MRI changes in MIE usually resolve dramatically on discontinuation of treatment, and thus MIE with brain cystic lesions has rarely been reported. A 49-year-old woman was treated for a lumbar abscess with oral administration of $1.5 \mathrm{~g} /$ day of metronidazole for 4 months. Neurological examination revealed aphasia, apraxia, agraphia, cerebellar ataxia, and cognitive impairment. On brain MRI, diffusion-weighted imaging and the apparent diffusion coefficients were consistent with cytotoxic edema in the corpus callosum and subcortical white matter, representing delayed huge cystic lesions. Thus, we should be aware of MIE with irreversible brain lesions.
\end{abstract}

Keywords: Metronidazole; Encephalopathy; Cystic lesion

\section{Image Description}

Metronidazole-induced encephalopathy (MIE) is a rare clinical condition resulting from long-term use of metronidazole. The symptoms and brain MRI changes in MIE usually resolve dramatically on discontinuation of treatment, and thus MIE with brain cystic lesions has rarely been reported [1,2].

A 49-year-old woman was treated for a lumbar abscess with oral administration of $1.5 \mathrm{~g}$ /day of metronidazole for 4 months. Neurological examination revealed aphasia, apraxia, agraphia, cerebellar ataxia, and cognitive impairment.

On brain MRI, symmetrical hyperintense lesions were observed in the bilateral middle cerebellar peduncles (arrows in A, D and G), bilateral cerebellar dentate nuclei (arrowheads in A, D and G), genu of the corpus callosum (arrows in B and E), splenium of the corpus callosum (arrowheads in B and E), and subcortical white matter (arrows in $\mathrm{C}$ and $\mathrm{F}$ ) on FLAIR imaging, diffusion-weighted imaging (DWI), and apparent diffusion coefficient (ADC) mapping, before withdrawal. Low intensity lesions are observed in the genu of the corpus callosum (arrow in $\mathrm{H}$ ) and subcortical white matter (arrows in I) on ADC maps, before withdrawal. Although follow-up MRI (1 month after withdrawal) of the cerebellum revealed the abnormal intensities in the middle cerebellar peduncles and cerebellar dentate nuclei had disappeared (J), huge cystic lesions were observed in the genu of the corpus callosum (arrow in $\mathrm{K}$ ) and subcortical white matter (arrows in L), suggesting that cytotoxic edema had occurred. Thus, we should be aware of MIE with irreversible brain lesions.

\section{References}

1. Erdener SE, Kansu T, Arsava EM, Dericioglu N (2013) Brain MRI evolution of metronidazole intoxication. Neurology 19: 1816-1817.

2. Furukawa S, Yamamoto T, Sugiyama A, Ohira K, Aotsuka Y, et al. (2015) Metronidazole-induced encephalopathy with contrast enhancing lesions on MRI. J Neurol Sci 352: 129-131. 
Citation: Koh K, Tsuchiya M, Takaki R, Togashi S, Nagasaka T (2016) Huge Brain Cystic Lesions Resulting from Metronidazole-Induced Encephalopathy. J Neurol Neurophysiol 7: i105. doi:10.4172/2155-9562.1000i105

Page 2 of 2

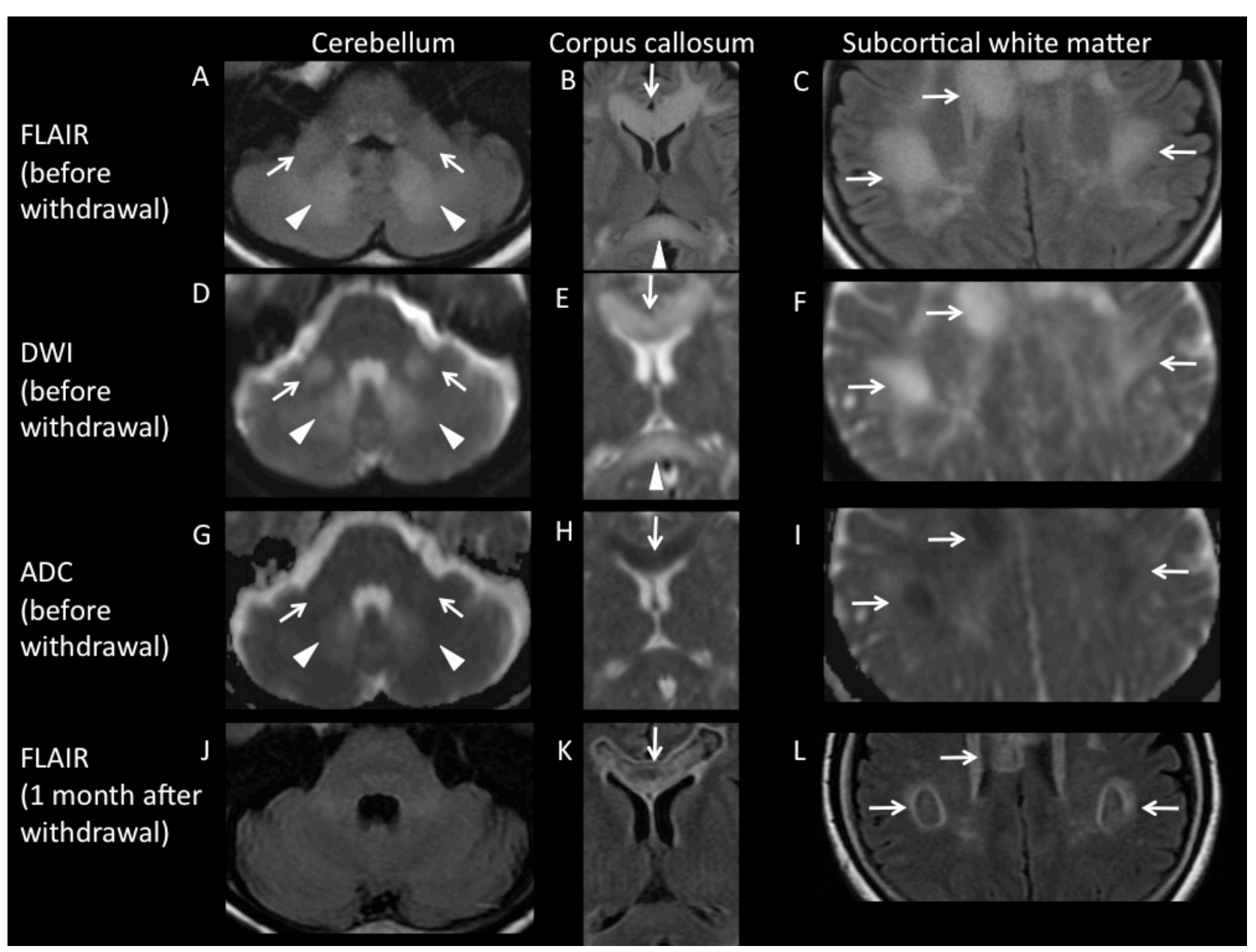

